\title{
Enhanced Lipid Peroxidation and Platelet Activation as Potential Contributors to Increased Cardiovascular Risk in the Low-HDL Phenotype
}

Natale Vazzana, MD; Antonina Ganci, MD; Angelo Baldassare Cefalù, MD; Stefano Lattanzio, BSc; Davide Noto, MD; Nicole Santoro, MD; Raoul Saggini, MD; Luca Puccetti, MD; Maurizio Averna, MD; Giovanni Davì, MD

Background-Low high-density lipoprotein (HDL) levels are major predictors of cardiovascular (CV) events, even in patients on statin treatment with low-density lipoprotein (LDL) at target. In animal models HDLs protect LDL from oxidation and blunt platelet activation. Our study aimed to examine whether HDL levels are related to in vivo oxidative stress and platelet activation, as determinants of atherothrombosis.

Methods and Results-Urinary 8-iso- $\mathrm{PGF}_{2 \alpha}$ and 11-dehydro- $\mathrm{TXB}_{2}$, in vivo markers of oxidative stress and platelet activation, respectively, were measured in 65 coronary heart disease (CHD) normocholesterolemic patients with $\mathrm{HDL} \leq 35 \mathrm{mg} / \mathrm{dL}$, and in 47 CHD patients with HDL $>35 \mathrm{mg} / \mathrm{dL}$. The 2 eicosanoids were also measured before and after an intensive exercise program in sedentary people $(n=18)$ and before and after fenofibrate treatment in otherwise healthy subjects with low HDL ( $n=10)$. Patients with $\mathrm{HDL} \leq 35 \mathrm{mg} / \mathrm{dL}$ showed significantly higher urinary 8-iso-PGF $2 \alpha$ (median [25th to 75th percentiles]: 289 [189 to 380 ] versus 216 [171 to 321] pg/mg creatinine, $P=0.019$ ) and 11-dehydro-TXB 2 (563 [421 to 767] versus 372 [249 to 465] pg/mg creatinine, $P=0.0001$ ) than patients with higher HDL. A direct correlation was found between urinary 8-iso-PGF $2 \alpha$ and 11-dehydro-TXB ${ }_{2}$ in the entire group of patients $(\rho=0.77, P<0.0001)$. HDL levels were inversely related to both 8 -iso-PGF $2 \alpha(\rho=-0.32, P=0.001)$ and 11-dehydro-TXB $2(\rho=-0.52, P<0.0001)$. On multiple regression, only 8 -iso- $\mathrm{PGF}_{2 \alpha}(\beta=0.68, P<0.0001)$ and $\mathrm{HDL}$ level $(\beta=-0.29$, $P<0.0001)$ were associated with urinary 11-dehydro-TXB $\mathrm{T}_{2}$ excretion, independent of sex, age, smoking, hypertension, diabetes, previous myocardial infarction, total cholesterol, LDL, and triglycerides. Both intensive exercise and fenofibrate treatment significantly reduced the 2 eicosanoids in healthy subjects, in parallel with an HDL increase.

Conclusions-A low HDL phenotype, both in CHD patients and in healthy subjects, is associated with increased lipid peroxidation and platelet activation. These data provide novel insight into the mechanisms linking low HDL with increased CV risk. ( $J$ Am Heart Assoc. 2013;2:e000063 doi: 10.1161/JAHA.113.000063)

Key Words: exercise - HDL cholesterol • oxidative stress • platelet

T igh-density lipoprotein (HDL) cholesterol is a major 1 independent risk factor for coronary heart disease (CHD). ${ }^{1}$

A low level of HDL cholesterol (HDL-C) is a powerful predictor of increased cardiovascular (CV) risk, ${ }^{2}$ and it

From the Department of Neuroscience and Imaging (R.S.) and Internal Medicine and Center of Excellence on Aging (N.V., S.L., N.S., G.D.), "G. d'Annunzio" University of Chieti, Chieti, Italy; Department of Internal Medicine, University of Palermo, Palermo, Italy (A.G., A.B.C., D.N., M.A.); Division of Hematology, Atherothrombosis Center, University of Siena, Siena, Italy (L.P.).

Correspondence to: Giovanni Davì, MD, Center of Excellence on Aging, “G. D’Annunzio" University Foundation, Via Colle dell'Ara, 66013 Chieti, Italy. E-mail: gdavi@unich.it

Received December 31, 2012; accepted February 25, 2013.

(C) 2013 The Authors. Published on behalf of the American Heart Association, Inc., by Wiley-Blackwell. This is an Open Access article under the terms of the Creative Commons Attribution Noncommercial License, which permits use, distribution and reproduction in any medium, provided the original work is properly cited and is not used for commercial purposes. remains a significant risk factor in people whose LDL cholesterol (LDL-C) is reduced to very low levels. ${ }^{3}$ Indeed, $\mathrm{HDL}-\mathrm{C}$ levels are predictive of major CV events in patients treated with statins, both when HDL-C is considered as a continuous variable and when subjects are stratified according to quintiles of HDL-C level. Even among subjects with LDL-C levels $<70 \mathrm{mg} / \mathrm{dL}$, those in the highest quintile of $\mathrm{HDL}-\mathrm{C}$ level are at less risk for major CV events than those in the lowest quintile. ${ }^{3}$ Furthermore, the ratio of LDL to HDL cholesterol is also highly predictive of the risk of major CV events.

In patients with established CV disease, residual CV risk persists despite the achievement of target LDL-C levels with statin therapy. Among patients with atherosclerotic CV disease and LDL-C levels $<70 \mathrm{mg} / \mathrm{dL}$, there is no incremental clinical benefit from the addition of niacin to statin therapy during a 36-month follow-up period, despite significant improvements in $\mathrm{HDL}-\mathrm{C}$ and triglyceride levels. ${ }^{4}$ 
Similarly, therapy with the cholesterol ester transfer protein (CEPT) inhibitor torcetrapib, despite a $72 \%$ increase in HDL-C levels, resulted in an increased risk of CV mortality and morbidity. ${ }^{5}$ However, the clinical failure of torcetrapib intervention trials has been related to "off-target" effects on neurohumoral regulation of blood pressure and increased risk of arrhythmias. In addition, CEPT inhibition generates large amounts of large HDL particles that are not optimally functional. ${ }^{6}$

Interventions aimed at increasing HDL-C levels prevent the progression of $\mathrm{CHD}^{7}$ In fact, niacin therapy, by increasing HDL levels, causes a significant regression of carotid intimamedia thickness when combined with a statin, as compared with ezetimibe. ${ }^{8}$ Interestingly, the incidence of major CV events is lower in the niacin group than in the ezetimibe group. ${ }^{8}$ Intensive statin regimen, either with atorvastatin or rosuvastatin, results in very low levels of LDL-C as well as a slight increase in HDL. The 2 regimens are similar in their ability to limit progression or induce regression of coronary disease evaluated by intravascular ultrasonography. ${ }^{9}$

In addition to its cholesterol-transporting properties, HDL favorably regulates endothelial cell phenotype, promoting the production of nitric oxide (NO) by upregulating endothelial NO synthase expression. Moreover, HDL's antithrombotic properties may be related to their abilities to attenuate the expression of tissue factor and selectins and to downregulate thrombin generation via the protein C pathway, thus directly and indirectly blunting platelet activation. ${ }^{10}$

HDL exhibits antioxidant activity by inhibition of LDL oxidation with a subsequent reduction of cellular uptake by the monocyte macrophage system. ${ }^{11}$ A normo-triglyceridemic, low HDL-C phenotype is characterized by elevated oxidative stress and HDL particles with attenuated antioxidant activity. ${ }^{12}$ Antioxidant activity of small dense HDL is deficient in type 2 diabetes, and it is linked to enhanced oxidative stress. ${ }^{13}$ The increased generation of reactive oxygen species may induce enhanced lipid peroxidation of cell-membrane phospholipids or circulating LDL, leading to the increased generation of F2-isoprostanes, a family of prostaglandin isomers produced from arachidonic acid by a mechanism catalyzed by free radicals. ${ }^{14,15} \mathrm{~F} 2$-isoprostanes can modulate the activation of platelets induced by low levels of other agonists. ${ }^{16}$ The consistent relationship between the rates of formation of F2-isoprostanes and thromboxane (TX) in several metabolic disorders ${ }^{16}$ suggests that TX-dependent platelet activation may be mediated, at least in part, by enhanced lipid peroxidation. ${ }^{17}$ This vicious cycle may be downregulated by a successful weight-loss program in obesity ${ }^{18}$ or by tight metabolic control obtained by insulin treatment in type 2 diabetes mellitus. ${ }^{19}$

We speculated that increased oxidative stress in CHD patients with a low-HDL phenotype could induce enhanced generation of 8-iso-prostaglandin $(\mathrm{PG}) \mathrm{F}_{2 \alpha}$ and other biologically active isoeicosanoids and that these compounds, in turn, could contribute to platelet activation in this setting. ${ }^{17}$ Therefore, in the present study, we set out to investigate whether 8-iso-PGF $2 \alpha$ formation is increased in CHD patients with lower HDL levels $(\leq 35 \mathrm{mg} / \mathrm{dL}$ ) when compared with CHD patients with higher HDL levels ( $>35 \mathrm{mg} / \mathrm{dL}$ ) and no significant differences in the other known $\mathrm{CV}$ risk factors and whether it correlates with the rate of $\mathrm{TXA}_{2}$ biosynthesis. Moreover, we examined the effects of 2 interventions aimed at increasing HDL levels, that is, fenofibrate treatment and 8 weeks of a standardized aerobic program, in otherwise healthy subjects with a low-HDL phenotype by assessing time-related changes in urinary 8 -iso- $\mathrm{PGF}_{2 \alpha}$ and in TXdependent platelet activation.

The results of the present study suggest that enhanced peroxidation of arachidonic acid to form biologically active isoprostanes may represent an important biochemical link between the low-HDL phenotype and persistent platelet activation in this setting.

\section{Methods}

\section{Design of the Studies}

The first study was a cross-sectional comparison of urinary F2-isoprostane 8-iso- $\mathrm{PGF}_{2 \alpha}$ and 11-dehydro-TXB ${ }_{2}$ (a major enzymatic metabolite of $\mathrm{TXA}_{2}$ ) of 65 normocholestoremic CHD patients with HDL-C levels $\leq 35 \mathrm{mg} / \mathrm{dL}$ (52 men, aged 62 [54 to 70] years) with 47 normocholestoremic CHD patients with HDL-C levels $>35 \mathrm{mg} / \mathrm{dL}$ (30 men, aged 65 [57 to 68] years). CHD incidents (including angina pectoris, exercise stress testing positive for ischemia, and acute myocardial infarction in the previous 24 months) were recorded from the outpatient database or from hospital discharge diagnoses. All subjects were attending the Lipid Clinics of the University of Palermo School of Medicine. CV risk factors such as smoking, hypertension, and diabetes were equally represented in the 2 groups (Table 1).

None of the patients had taken any drugs known to affect lipid metabolism or platelet function for $\geq 2$ weeks before the start of the study because they were unwilling to do so. Patients with renal insufficiency or proteinuria (by serum creatinine level and urinalysis), altered hepatic function (by liver enzymes), or alcohol abuse were excluded.

All subjects were studied as outpatients after a 12-hour fast. Blood samples were obtained for the following measurements: total cholesterol, triglycerides, HDL cholesterol. Each patient performed a 12-hour overnight urine collection immediately before blood sampling. Urine samples were supplemented with the antioxidant 4-hydroxy-tempo $(1 \mathrm{mmol} / \mathrm{L})$ and stored at $-20^{\circ} \mathrm{C}$ until extraction. 
Table 1. Clinical Characteristics of CHD Patients

\begin{tabular}{|c|c|c|c|}
\hline Variables & HDL-C $\leq 35 \mathrm{mg} / \mathrm{dL}(\mathrm{n}=65)$ & HDL-C $>35 \mathrm{mg} / \mathrm{dL}(\mathrm{n}=47)$ & $P$ Value ${ }^{*}$ \\
\hline Age, y & 62 (54 to 70 ) & 65 (57 to 68 ) & 0.439 \\
\hline Male sex, n (\%) & $52(80)$ & $30(64)$ & 0.09 \\
\hline Total cholesterol, mg/dL & 155 (133 to 180 ) & 166 (152 to 182 ) & 0.063 \\
\hline Triglycerides, mg/dL & 84 (55 to 153 ) & 85 (62 to 116 ) & 0.874 \\
\hline $\mathrm{HDL}-\mathrm{C}, \mathrm{mg} / \mathrm{dL}$ & 28 (25 to 32 ) & 43 (39 to 47$)$ & $<0.001$ \\
\hline LDL-C, mg/dL & 110 (89 to 122$)$ & 99 (87 to 113$)$ & 0.153 \\
\hline Cigarette smoking, n (\%) & $21(32)$ & $13(20)$ & 0.749 \\
\hline Arterial hypertension, $\mathrm{n}(\%)$ & $36(55)$ & $28(43)$ & 0.804 \\
\hline Type 2 diabetes, n (\%) & $26(40)$ & $14(22)$ & 0.36 \\
\hline Previous Ml, n (\%) & $24(37)$ & $16(25)$ & 0.90 \\
\hline
\end{tabular}

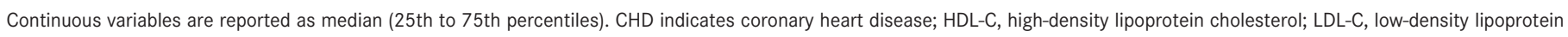
cholesterol; MI, myocardial infarction.

*By Mann-Whitney, chi-square, or Fisher exact tests, as appropriate.

The study was approved by the local Ethics Committee, and all patients gave their written informed consent to participate in the study.

A second study was performed to investigate the effects of regular high-amount, high-intensity aerobic exercise on in vivo lipid peroxidation and platelet activation in 18 healthy sedentary people with low HDL-C levels. We evaluated whether changes in HDL-C levels, induced by the exercise program, were related to changes in the 2 eicosanoids. Each participant completed an 8-week standardized aerobic highamount, high-intensity training program. Urinary 8 -iso-PGF $2 \alpha$ and 11-dehydro- $\mathrm{TXB}_{2}$ excretion rates had been measured before and after this intervention.

After giving written informed consent, 18 subjects (13 men, aged 50 [48 to 66] years) had been enrolled in the study. Subjects were enrolled if they had a sedentary lifestyle (regular aerobic exercise $<3$ times/weeks and $<20$ minutes/ session), had a sedentary occupation, and had a baseline HDL cholesterol concentration $<55 \mathrm{mg} / \mathrm{dL} \quad(<1.4 \mathrm{mmol} / \mathrm{L})$. Exclusion criteria included obesity (body mass index [BMI] $\geq 30 \mathrm{~kg} / \mathrm{m}^{2}$ ), diagnosis of diabetes mellitus, poorly controlled hypertension or hypercholesterolemia, pregnancy, impaired liver or renal function, previous vascular event (myocardial infarction, stroke, transient ischemic attack), or other medical conditions that would preclude vigorous exercise, and treatment with nonsteroidal anti-inflammatory drugs (NSAIDs), antioxidant supplements, anticoagulants, or antiplatelet drugs.

Each participant completed an 8-week standardized aerobic training program. The exercise training involved 2 sessions per week of supervised exercise on a cycle ergometer (Monark 915E, Vansbro, Sweden). The exercise prescription in the exercise group was high-amount, high-intensity exercise for 55 minutes per session, the caloric equivalent of jogging $\approx 20$ miles $(32.0 \mathrm{~km}$ ) per week at $60 \%$ to $75 \%$ of peak oxygen consumption. ${ }^{20}$ There was an initial period of 1 month during which the amount and intensity of exercise were gradually increased, followed by 8 weeks at the appropriate exercise prescription. Participants started at $55 \%$ of their baseline $\mathrm{VO}_{2}$ max for 45 minutes per session and progressed in intensity or duration every week according to a standardized protocol until achieving the standards scheduled for the training program ( 55 minutes at $75 \%$ of baseline $\mathrm{VO}_{2}$ max). All exercise sessions were verified by direct supervision or by heart rate monitors that provided recorded data (Polar Electro). Daily energetic consumption was monitored by metabolic armband (Sensewear Pro3).

A third study was performed to investigate the effects of 3-month administration of fenofibrate ( $145 \mathrm{mg} /$ day) to 10 subjects ( 7 men, aged 58 [55 to 60] years) with a low-HDL phenotype and triglycerides $>200 \mathrm{mg} / \mathrm{dL}$, otherwise healthy, previously unresponsive to an appropriate dietary regimen (checked regularly by a qualified nutritionist). The 10 subjects were allocated to continue the previous diet plus fenofibrate for 3 months.

All patients had a positive familial history for CV events younger than age 65 . Exclusion criteria included obesity (BMI $\geq 30 \mathrm{~kg} / \mathrm{m}^{2}$ ), diabetes mellitus, poorly controlled hypertension or hypercholesterolemia, pregnancy, impaired liver or renal function, previous vascular event (myocardial infarction, stroke, transient ischemic attack), and treatment with NSAIDs, antioxidant supplements, anticoagulants, or antiplatelet drugs.

This study was approved by the local Ethics Committee, and all patients gave their written informed consent to participate in the study. 
Assays

All participants were instructed to perform an overnight urine collection and underwent a fasting blood sample drawn the following morning. Plasma, serum, and urine were stored in aliquots at $-20^{\circ} \mathrm{C}$ until used for the various analyses.

Urinary 8-iso-PGF ${ }_{2 \alpha}$ and 11-dehydro-TXB ${ }_{2}$ excretion rates were measured by previously described radioimmunoassay methods. ${ }^{21,22}$ These methods have been validated by comparison with gas chromatography/mass spectrometry, as detailed elsewhere. ${ }^{21,22}$

Total cholesterol, triglycerides, high-density lipoprotein cholesterol (HDL-C), and low-density lipoprotein cholesterol (LDL-C) concentrations were measured as previously described. $^{23}$

\section{Statistical Analysis}

The Kolmogorov-Smirnov test was used to determine whether each variable had a normal distribution. When necessary, logtransformation was used to normalize the data, or appropriate nonparametric tests were used. Comparisons of baseline data between the groups were performed by chi-square statistics, Fisher exact tests, or Mann-Whitney $U$ tests.

The differences between baseline and posttreatment values were analyzed with the Wilcoxon signed rank test. The association of eicosanoid measurements with other biochemical parameters was assessed by the Spearman rank correlation test.

Stepwise multiple linear regression analysis was performed to assess variables independently associated with urinary 11-dehydro- $\mathrm{TXB}_{2}$ excretion rates, with logarithmically transformed data for this analysis and forward selection of variables. Covariates included in the multiple regression models were selected on the basis of their significance in univariate analysis and their clinical relevance to the outcome of interest, as reported from other studies. They included sex, age, smoking, hypertension, diabetes, previous myocardial infarction, total cholesterol, LDL-C, HDL-C, triglycerides, and urinary 8-iso-PGF $2 \alpha$.

All values are reported as median (interquartile range [IQR]). $P$ values $<0.05$ were regarded as statistically significant. All tests were 2-tailed, and analyses were performed using the SPSS (version 16.0; APSS, Chicago, IL), statistical package.

\section{Results}

The baseline characteristics of the $112 \mathrm{CHD}$ patients are detailed in Table 1. The 2 groups of patients (CHD with HDL-C levels $\leq 35$ and $>35 \mathrm{mg} / \mathrm{dL}$ ) were comparable both for sex distribution and for age. No statistically significant differences were found between the 2 groups of patients for CV risk factors.

Urinary excretion rate of the F2-isoprostane 8-iso- $\mathrm{PGF}_{2 a}$ was significantly $(P=0.019)$ higher in the CHD patients with $\mathrm{HDL} \leq 35 \mathrm{mg} / \mathrm{dL}$ than in the patients with higher HDL levels: 289 (189 to 380 ) versus 216 (171 to 321 ) pg/mg creatinine (Figure 1A). Moreover, patients with $\mathrm{HDL} \leq 35 \mathrm{mg} / \mathrm{dL}$ had a significantly $(P=0.0001)$ higher excretion rate of 11-dehydro$\mathrm{TXB}_{2}$, an index of in vivo platelet activation, than did patients with higher HDL levels: 563 (421 to 767) versus 372 (249 to 465) $\mathrm{pg} / \mathrm{mg}$ creatinine (Figure 1B).

As depicted in Figure 2, a highly statistically significant direct correlation was found between the rates of excretion of 8-iso-PGF ${ }_{2 \alpha}$ and 11-dehydro-TXB ${ }_{2}$ in both groups of patients $(\rho=0.77, P<0.0001)$, suggesting a potential link between lipid peroxidation and platelet activation in this setting. In the

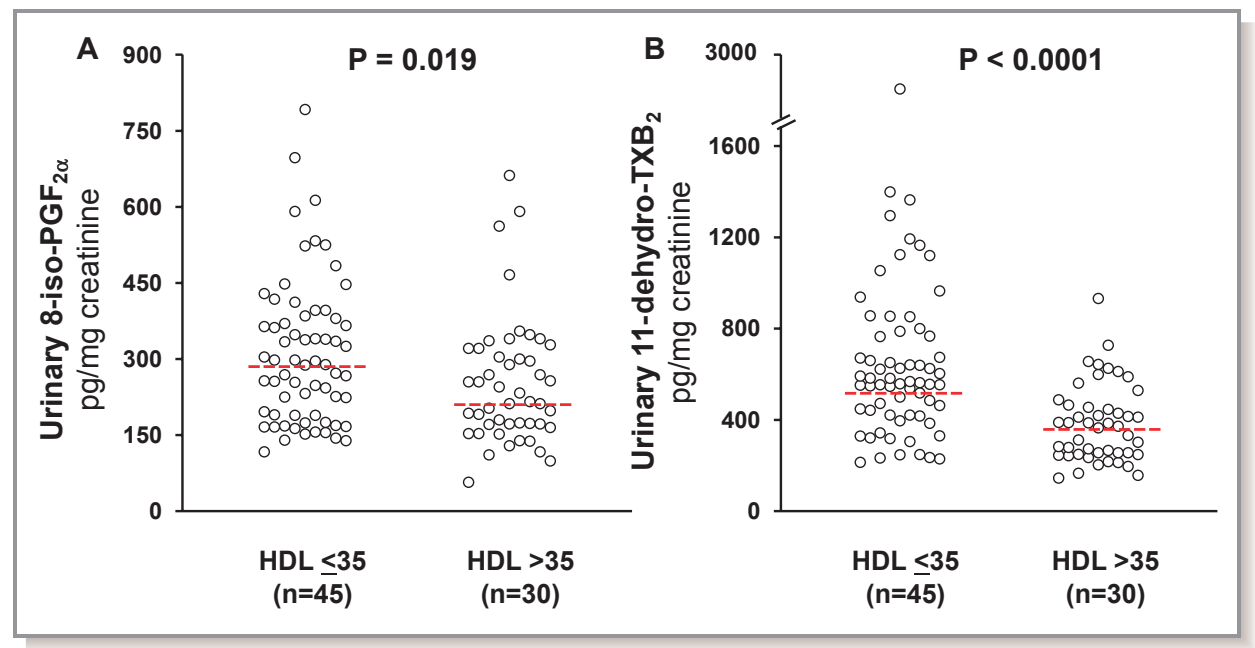

Figure 1. Urinary levels of 8-iso- $\mathrm{PGF}_{2 \alpha}(\mathrm{A})$ and 11-dehydro- $\mathrm{TXB}_{2}(\mathrm{~B})$ in normocholesterolemic $\mathrm{CHD}$ patients, according to $\mathrm{HDL}$ cholesterol levels. The horizontal dotted lines denote the median value for each study group. 8-iso-PGF $2 \alpha$ indicates 8-iso-prostaglandin $\mathrm{F}_{2 \alpha}$; 11-dehydro-TXB ${ }_{2}$, 11-dehydrothromboxane $\mathrm{B}_{2}$; $\mathrm{CHD}$, coronary heart disease; $\mathrm{HDL}$, high-density lipoprotein. 


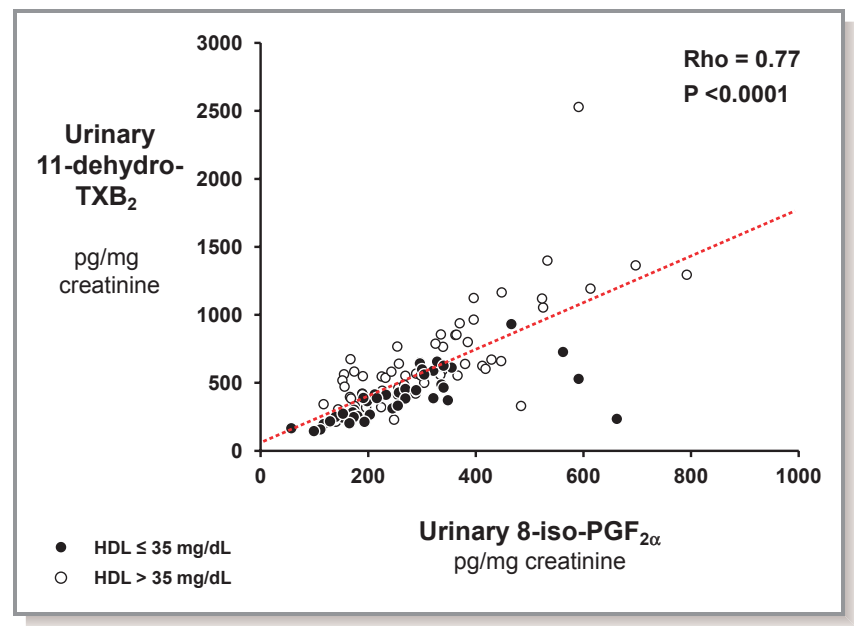

Figure 2. Correlation between urinary 8-iso- $\mathrm{PGF}_{2 \alpha}$ and 11-dehydro$\mathrm{TXB}_{2}$ in $\mathrm{CHD}$ patients with $\mathrm{HDL}$ cholesterol $\leq 35 \mathrm{mg} / \mathrm{dL}$ (closed circles) and with $\mathrm{HDL}>35 \mathrm{mg} / \mathrm{dL}$ (open circles). 8-iso- $\mathrm{PGF}_{2 \alpha}$ indicates 8-iso-prostaglandin $\mathrm{F}_{2 \alpha} ;$ 11-dehydro- $\mathrm{TXB}_{2}$, 11-dehydrothromboxane $\mathrm{B}_{2}$; $\mathrm{CHD}$, coronary heart disease; $\mathrm{HDL}$, high-density lipoprotein.

entire group of CHD patients, HDL level was inversely related to both 8-iso-PGF ${ }_{2 \alpha}(\rho=-0.32, P=0.001)$ and 11-dehydro$\operatorname{TXB}_{2}(\rho=-0.52, P<0.0001)$; see Figure 3 .

On multiple regression analysis, only urinary 8 -iso- $\mathrm{PGF}_{2 \alpha}$ ( $\beta=0.68, t=10.6, P<0.0001)$ and HDL level $(\beta=-0.29, t=-4.56$, $P<0.0001)$ were associated with urinary 11-dehydro-TXB ${ }_{2}$ levels, independently of sex, age, smoking, hypertension, diabetes, previous myocardial infarction, total cholesterol, LDL-C, and triglycerides.

\section{Effects of the Exercise Program}

We investigated the effects of an 8-week standardized highamount, high-intensity aerobic exercise on in vivo lipid peroxidation and platelet activation in 18 healthy sedentary people with low HDL cholesterol levels. Because weight loss may significantly affect in vivo lipid peroxidation and platelet activation, ${ }^{18}$ subjects were given the recommendation of maintaining body weight during the 2-month period, and thus they had a small but not significant weight gain.

Exercise training had no significant effect on total cholesterol or LDL-C concentrations. There was a statistically significant increase $(P=0.041)$ of $\mathrm{HDL}-\mathrm{C}$ levels, from 47.5 (42.5 to 49.0 ) to 52.5 (45.0 to 61.3$) \mathrm{mg} / \mathrm{dL}$, after the highamount, high-intensity training period. There was also a significant reduction in triglyceride concentration (from 119 [92 to 177] to 95 [77 to 118$] \mathrm{mg} / \mathrm{dL}, P=0.025$ ).

The 8-week exercise program was associated with a significant reduction in the urinary excretion rate of both 8-iso-PGF $2 \alpha$ and 11-dehydro-TXB 2 (by $25 \%$ [9.3\% to $58 \%$ ] and $29 \%$ [20\% to $40 \%$ ], respectively, versus baseline; $P<0.0001$; Figure $4 \mathrm{~A}$ and $4 \mathrm{~B})$.

Statistically significant inverse correlations were found between HDL-C and 11-dehydro-TXB 2 levels $(\rho=-0.38$, $P=0.023$ ) over the 8 -week training period by pooling the data obtained before and after exercise (Figure $4 \mathrm{C}$ ).

\section{Effects of Fenofibrate}

We next examined the effects of 3 months of administration of fenofibrate (145 mg/day) on the urinary excretion of 8-iso$\mathrm{PGF}_{2 \alpha}$ and 11-dehydro-TXB ${ }_{2}$ in subjects with a low-HDL phenotype and triglycerides $>200 \mathrm{mg} / \mathrm{dL}$ but otherwise healthy to test the hypothesis of a cause-effect relationship between a low-HDL phenotype and enhanced lipid peroxidation and platelet activation in the setting of healthy people with low HDL-C levels.

After fenofibrate treatment, HDL-C levels were significantly increased, by $14 \%$ (5.0\% to $25 \%$; $P=0.007)$, whereas triglyceride levels were significantly decreased, by $41 \%$ (38\% to $46 \%$; $P=0.005$ ). Despite this, both urinary 8 -iso- $P_{G F} F_{2 \alpha}$ and

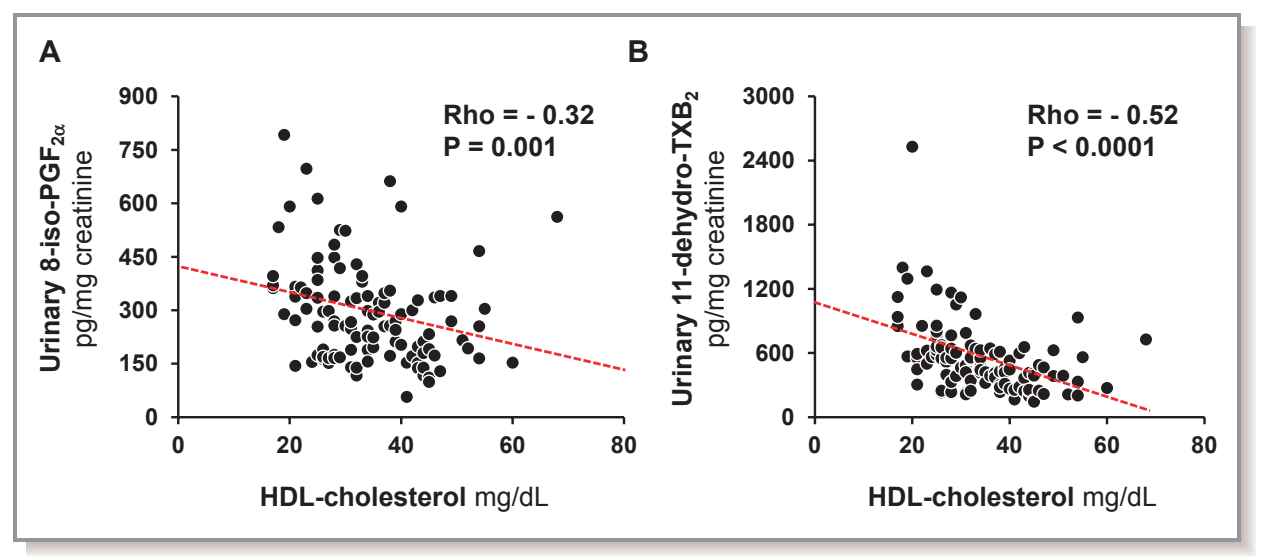

Figure 3. Correlations between HDL cholesterol and urinary 8-iso-PGF ${ }_{2 \alpha}(\mathrm{A})$ and 11-dehydro-TXB $(\mathrm{B})$ in $\mathrm{CHD}$ patients. HDL indicates highdensity lipoprotein; 8-iso-PGF $\mathrm{PG}_{2 \alpha}$, 8-iso-prostaglandin $\mathrm{F}_{2 \alpha} ; 1$ 1-dehydro- $\mathrm{TXB}_{2}$, 11-dehydrothromboxane $\mathrm{B}_{2}$; $\mathrm{CHD}$, coronary heart disease. 


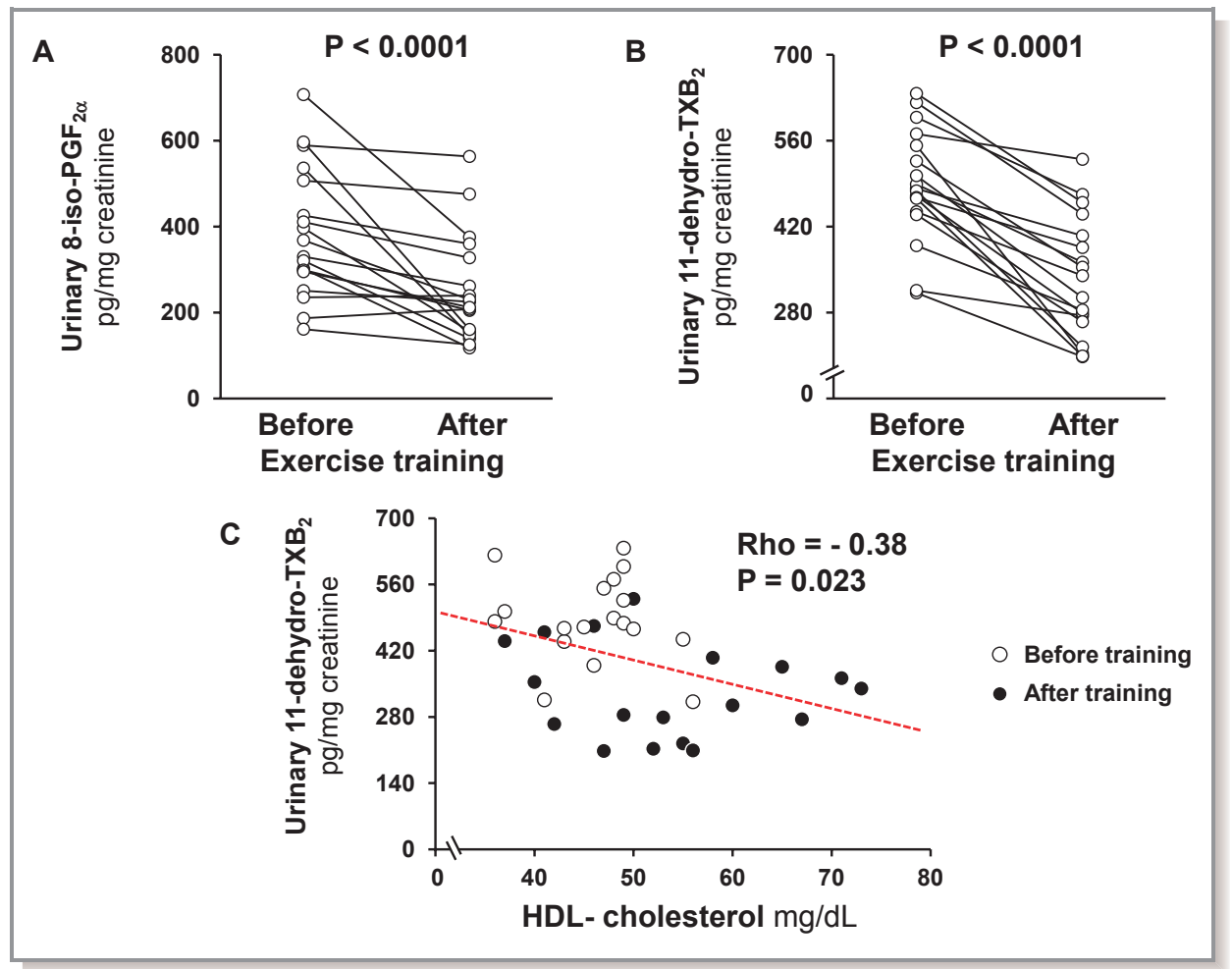

Figure 4. Urinary levels of 8-iso-PGF $2 \alpha(\mathrm{A})$ and 11-dehydro- $\mathrm{TXB}_{2}(\mathrm{~B})$ in 18 healthy sedentary subjects before and after an aerobic high-amount, high-intensity training program. $\mathrm{C}$, Correlation between urinary 8 -iso- $\mathrm{PGF}_{2 \alpha}$ and 11-dehydro-TXB $\mathrm{B}_{2}$ before (open circles) and after (closed circles) exercise training. 8-iso- $\mathrm{PGF}_{2 \alpha}$ indicates 8-iso-prostaglandin $\mathrm{F}_{2 \alpha} ; 11$-dehydro- $\mathrm{TXB}_{2}$, 11-dehydrothromboxane $\mathrm{B}_{2}$; HDL, high-density lipoprotein.

11-dehydro- $\mathrm{TXB}_{2}$ excretion rates were not significantly changed in the whole group. However, in the 5 subjects in whom fenofibrate induced an increase of HDL cholesterol level above the median, we observed a significant reduction in urinary 8 -iso-PGF ${ }_{2 \alpha}$ and 11-dehydro-TXB ${ }_{2}$ excretion rates, by $39 \%(27 \%$ to $45 \%)$ and $70 \%(45 \%$ to $72 \%)$, respectively $(P=0.043)$ in comparison with no significant modifications obtained in the 5 subjects who had an increase of HDL cholesterol below the median (Figure 5). Moreover, in the former group of subjects, all individual values of urinary 8-iso-PGF ${ }_{2 \alpha}$ and 11-dehydro-TXB ${ }_{2}$ levels fell within the range of those of healthy subjects. ${ }^{18}$ Finally, the changes in 8-iso-PGF ${ }_{2 \alpha}$ levels were also associated with the reduction in urinary 11-dehydro- $\mathrm{TXB}_{2}$ levels because a statistically significant correlation was found between the rates of excretion of these eicosanoids throughout the range of values measured at baseline and after 3 months of administration of $145 \mathrm{mg}$ of fenofibrate $(\rho=0.77, P=0.009)$.

\section{Discussion}

Low HDL-C is an independent CV risk factor, and the increase of HDL-C of only $1 \mathrm{mg} / \mathrm{dL}$ leads to a risk reduction of $2 \%$ to $3 \%$. ${ }^{1,2}$ Despite aggressive LDL-C lowering with statin therapy, a residual risk of $\mathrm{CV}$ morbidity and mortality still occurs in a significant portion of subjects. ${ }^{3,4}$ There is compelling evidence in animal models that increasing HDL levels is antiatherogenic. ${ }^{1}$ Therefore, raising HDL-C was thought of as a strategy for reducing the residual CV risk. However, a recent systematic review and meta-regression analysis of randomized controlled trials ${ }^{24}$ has suggested that increasing the amount of HDL-C does not reduce the risk of coronary heart disease events and mortality.

HDLs have several protective properties that are independent of their involvement in cholesterol metabolism. They have antiatherogenic properties that reduce oxidation and vascular inflammation and improve endothelial function, thus promoting endothelial repair. Among antioxidant properties of $\mathrm{HDL}$, the transfer of oxidized lipids from LDL to HDL, including oxidized phospholipids such as hydroperoxides and F2-isoprostanes, facilitates subsequent degradation of these products by HDL enzymes or by delivery to the liver for degradation, thus preventing pathological activities of these molecules. Normal functional HDL has high anti-oxidant potential, but when these antioxidant functions are overwhelmed by pathological processes, HDL is converted into a dysfunctional particle characterized by decreased anti-inflammatory and antioxidant properties. ${ }^{25}$

In vitro studies show that HDL inhibits agonist-stimulated platelet aggregation. ${ }^{26}$ This inhibitory effect is mediated by 


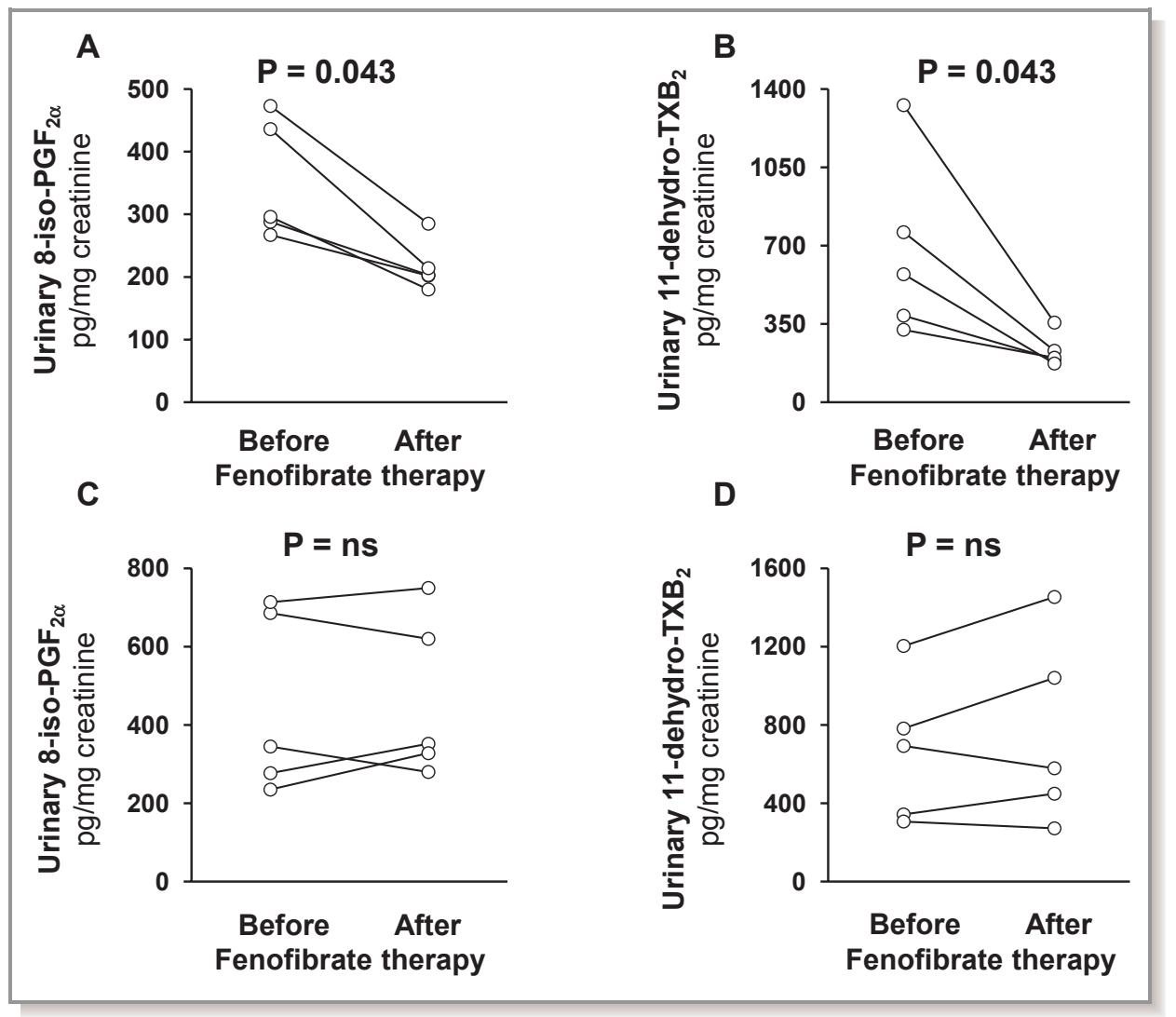

Figure 5. Urinary levels of 8-iso- $\mathrm{PGF}_{2 \alpha}$ and 11-dehydro- $\mathrm{TXB}_{2}$ in 10 healthy individuals with low HDL cholesterol and hypertriglyceridemia before and after a 3-month fenofibrate (145 $\mathrm{mg} /$ day) treatment. A and B, Subjects who had an increase in HDL cholesterol above the median. C and $\mathrm{D}$, Subjects who had an increase in HDL cholesterol below the median. 8-iso- PGF $_{2 \alpha}$ indicates 8-iso-prostaglandin $\mathrm{F}_{2 \alpha}$; 11-dehydro-TXB ${ }_{2}$, 11-dehydrothromboxane $B_{2}$; ns, not significant; $H D L$, high-density lipoprotein.

scavenger receptor type $\mathrm{B} 1$ and/or the apolipoprotein $\mathrm{E}$ receptor apoER2/LRP8 and is linked to the induction of intracellular signaling cascades encompassing stimulation of protein kinase $\mathrm{C}$, cytoplasmatic alkalization, and generation of nitric oxide. Moreover, the infusion of Apo A-1 Milano (a protective variant of the major protein constituent of $\mathrm{HDL}$ ) into rats inhibits platelet aggregation. ${ }^{10}$

However, no evidence has been provided for the association between a low-HDL phenotype and in vivo platelet activation as well as in vivo lipid peroxidation.

Biochemical evidence of increased platelet activation in vivo was obtained through noninvasive measurements of TX metabolite excretion ${ }^{22,27}$ that avoided artifactual platelet activation during and after blood sampling. Although we did not measure platelet aggregation in our study, it should be emphasized that the ex vivo measurement of platelet responses to various agonists represents an index of functional capacity that by no means reflects the extent of platelet activation in vivo. ${ }^{27}$ The excretion rate of the major enzymatic metabolite of $\mathrm{TXA}_{2}, 11$-dehydro-TXB ${ }_{2}$, has been validated as a reliable and noninvasive, integrated index of in vivo platelet activation. ${ }^{17}$
Moreover, increased generation of reactive oxidant species can induce enhanced lipid peroxidation of cell-membrane phospholipids or circulating LDL, leading to increased generation of F2-isoprostanes, bioactive compounds produced from arachidonic acid by a free radical-catalyzed mechanism of lipid peroxidation. ${ }^{28}$ Activated platelets are also a source of reactive oxidant species (by NADPH oxidase) ${ }^{29}$ and might contribute to isoprostane generation by COX-dependent mechanisms. ${ }^{30}$ It has been proposed that F2-isoprostanes might transduce the effects of oxidant stress associate with complex metabolic disorders into specialized forms of cellular activation. In particular, 8-iso-PGF $2 \alpha$ might be able to modify platelet adhesive reactions and platelet activation in vitro by low concentrations of agonists. ${ }^{16}$

In the present report, we performed a series of studies to test the hypothesis that persistent in vivo platelet activation maybe related, at least in part, to enhanced urinary excretion of the F2-isoprostane 8-iso-PGF $2 \alpha$ as a marker of in vivo lipid peroxidation in the setting of a low-HDL phenotype.

In the first study, we performed a cross-sectional evaluation of the 2 eicosanoids in normocholesterolemic CHD patients, and we found that the formation and urinary 
excretion of 8-iso-PGF ${ }_{2 \alpha}$ was elevated in the vast majority of a relatively large group of patients with HDL-C levels $<35 \mathrm{mg} / \mathrm{dL}$ in comparison with a group of CHD patients with higher HDL-C levels, carefully characterized for other variables potentially influencing lipid peroxidation (see Table 1). ${ }^{28}$ Moreover, we have identified a novel mechanism through which low HDL-C levels may affect CV morbidity and mortality, that is, TX-dependent platelet activation. In fact, low HDL-C levels in CHD patients were associated with a significantly higher rate of TX metabolite excretion than measured in CHD patients with higher HDL-C levels. The consistent linear relationship between the excretion of rates of 8-iso-PGF ${ }_{2 \alpha}$ and 11-dehydro- $\mathrm{TXB}_{2}$ demonstrated in our study confirms and extends previous findings in subjects with other $\mathrm{CV}$ risk factors such diabetes mellitus or hypercholesterolemia. ${ }^{17}$

Further evidence for a cause-and-effect relationship between low HDL-C level and persistent platelet activation was obtained through 2 interventional studies: the first in sedentary people, the second in subjects with low HDL-C levels but otherwise healthy.

When we performed a study with a high-intensity, highamount exercise program for 2 months for sedentary people, we observed significant decreases in both TX-dependent platelet activation (11-dehydro- $\mathrm{TX}_{2}$ ) and lipid peroxidation (8-iso-PGF ${ }_{2 \alpha}$ ), with an inverse correlation between the TX metabolite and HDL level. Our data are in accordance with a recent study of adolescents with metabolic syndrome reporting that a physical activity program induced amelioration of HDL levels as well as of markers of oxidative stress. ${ }^{31}$

In healthy subjects with low HDL-C and high triglycerides, the reduction in both lipid peroxidation and TX-dependent platelet activation was proportional to the effect of fenofibrate on HDL levels. In fact, HDL-C increase above the median led to normalization of the 2 noninvasive indexes of platelet activation and lipid peroxidation. This is consistent with the finding that age-related changes in middle-aged nonobese men show a simultaneous decrease in HDL-C levels as well as plasma oxidative stress markers (8-iso-PGF $2 \alpha$ and malondialdehyde). ${ }^{32}$

The results of our study suggest that markers of lipid peroxidation and TX-dependent platelet activation may be useful synergistically with measurement of dysfunctional HDLs to improve CV risk stratification in patients with low HDL-C.

Several limitations of the intervention studies should be acknowledged. These include lack of randomization and small sample size of the 2 interventional studies. Despite these limitations, our findings may have important clinical implications for primary and secondary prevention in patients with a low HDL phenotype, providing novel insight into the mechanisms linking low HDL and occurrence of $\mathrm{CV}$ disease. In fact, within the limits of our relatively small mechanistic studies with biochemical end points, our results suggest that a substantial reduction in TX-dependent platelet activation can be achieved by a significant increase in HDL levels obtained through different tools (exercise and fenofibrate). For subjects who fail to achieve a substantial HDL-C increase, low-dose aspirin may be considered as an option after evaluating the potential benefit and hemorrhagic risk of the individual patient. ${ }^{33}$

\section{Sources of Funding}

This study was supported by grants from the European Commission (EICOSANOX Integrated Project 005033).

\section{Disclosures}

None.

\section{References}

1. Ashen MD, Blumenthal RS. Clinical practice. Low HDL cholesterol levels. $N$ Engl J Med. 2005;353:1252-1260.

2. Sharrett AR, Ballantyne CM, Coady SA, Heiss G, Sorlie PD, Catellier D, Patsch W; Atherosclerosis Risk in Communities Study Group. Coronary heart disease prediction from lipoprotein cholesterol levels, triglycerides, lipoprotein(a), apolipoproteins $\mathrm{A}-\mathrm{I}$ and $\mathrm{B}$, and $\mathrm{HDL}$ density subfractions: the Atherosclerosis Risk in Communities (ARIC) Study. Circulation. 2001;104:1108-1113.

3. Barter P, Gotto AM, LaRosa JC, Maroni J, Szarek M, Grundy SM, Kastelein JJ, Bittner V, Fruchart JC; Treating to New Targets Investigators. HDL cholesterol, very low levels of LDL cholesterol, and cardiovascular events. N Engl J Med. 2007;357:1301-1310.

4. AIM-HIGH Investigators, Boden WE, Probstfield JL, Anderson T, Chaitman BR, Desvignes-Nickens P, Koprowicz K, McBride R, Teo K, Weintraub W. Niacin in patients with low HDL cholesterol levels receiving intensive statin therapy. N Engl J Med. 2011;365:2255-2267.

5. Barter PJ, Caulfield M, Eriksson M, Grundy SM, Kastelein JJ, Komajda M, LopezSendon J, Mosca L, Tardif JC, Waters DD, Shear CL, Revkin JH, Buhr KA, Fisher MR, Tall AR, Brewer B; ILLUMINATE Investigators. Effects of torcetrapib in patients at high risk for coronary events. N Engl J Med. 2007;357:2 109-2122.

6. Goldberg AS, Hegele RA. Cholesteryl ester transfer protein inhibitors for dyslipidemia: focus on dalcetrapib. Drug Des Devel Ther. 2012;6:251-259.

7. Whitney EJ, Krasuski RA, Personius BE, Michalek JE, Maranian AM, Kolasa MW, Monick E, Brown BG, Gotto AM Jr. A randomized trial of a strategy for increasing high-density lipoprotein cholesterol levels: effects on progression of coronary heart disease and clinical events. Ann Intern Med. 2005;142:95-104.

8. Kastelein JJ, Bots ML. Statin therapy with ezetimibe or niacin in high-risk patients. N Engl J Med. 2009;361:2180-2183.

9. Nicholls SJ, Ballantyne CM, Barter PJ, Chapman MJ, Erbel RM, Libby P, Raichlen JS, Uno K, Borgman M, Wolski K, Nissen SE. Effect of two intensive statin regimens on progression of coronary disease. N Engl J Med. 2011;365:20782087.

10. Mineo C, Deguchi H, Griffin JH, Shaul PW. Endothelial and antithrombotic actions of HDL. Circ Res. 2006;98:1352-1364.

11. Navab M, Ananthramaiah GM, Reddy ST, Van Lenten BJ, Ansell BJ, Fonarow GC, Vahabzadeh K, Hama S, Hough G, Kamranpour N, Berliner JA, Lusis AJ, Fogelman AM. The oxidation hypothesis of atherogenesis: the role of oxidized phospholipids and HDL. J Lipid Res. 2004;45:993-1007.

12. Kontush A, de Faria EC, Chantepie S, Chapman MJ. A normotriglyceridemic, low HDL-cholesterol phenotype is characterised by elevated oxidative stress and $\mathrm{HDL}$ particles with attenuated antioxidative activity. Atherosclerosis. 2005; 182:277-285.

13. Nobécourt E, Jacqueminet S, Hansel B, Chantepie S, Grimaldi A, Chapman MJ, Kontush A. Defective antioxidative activity of small dense HDL3 particles in type 2 diabetes: relationship to elevated oxidative stress and hyperglycaemia. Diabetologia. 2005;48:529-538.

14. Morrow JD, Hill KE, Burk RF, Nammour TM, Badr KF, Roberts LJ II. A series of prostaglandin F2-like compounds are produced in vivo in humans by a non- 
cyclooxygenase, free radical-catalyzed mechanism. Proc Natl Acad Sci USA. 1990;87:9383-9387.

15. Morrow JD. Quantification of isoprostanes as indices of oxidant stress and the risk of atherosclerosis in humans. Arterioscler Thromb Vasc Biol. 2005;25: 279-286.

16. Pratico D, Smyth EM, Violi F, FitzGerald GA. Local amplification of platelet function by 8-Epi prostaglandin F2alpha is not mediated by thromboxane receptor isoforms. J Biol Chem. 1996;271:14916-14924.

17. Davì G, Patrono C. Platelet activation and atherothrombosis. N Eng/ J Med. 2007;357:2482-2494.

18. Davì G, Guagnano MT, Ciabattoni G, Basili S, Falco A, Marinopiccoli M, Nutini $\mathrm{M}$, Sensi S, Patrono C. Platelet activation in obese women: role of inflammation and oxidant stress. JAMA. 2002;288:2008-2014.

19. Davì G, Ciabattoni G, Consoli A, Mezzetti A, Falco A, Santarone S, Pennese E, Vitacolonna E, Bucciarelli T, Costantini F, Capani F, Patrono C. In vivo formation of 8-iso-prostaglandin f2alpha and platelet activation in diabetes mellitus: effects of improved metabolic control and vitamin E supplementation. Circulation. 1999;99:224-229.

20. Kraus WE, Houmard JA, Duscha BD, Knetzger KJ, Wharton MB, McCartney JS, Bales CW, Henes S, Samsa GP, Otvos JD, Kulkarni KR, Slentz CA. Effects of the amount and intensity of exercise on plasma lipoproteins. $N$ Engl J Med. 2002;347:1483-1492.

21. Wang Z, Ciabattoni G, Créminon C, Lawson J, Fitzgerald GA, Patrono C, Maclouf J. Immunological characterization of urinary 8-epi-prostaglandin F2 alpha excretion in man. J Pharmacol Exp Ther. 1995;275:94-100.

22. Ciabattoni G, Pugliese F, Davì G, Pierucci A, Simonetti BM, Patrono C. Fractional conversion of thromboxane $\mathrm{B} 2$ to urinary 11-dehydrothromboxane B2 in man. Biochim Biophys Acta. 1989;992:66-70.

23. Davì G, Averna M, Catalano I, Barbagallo C, Ganci A, Notarbartolo A, Ciabattoni $\mathrm{G}$, Patrono C. Increased thromboxane biosynthesis in type lla hypercholesterolemia. Circulation. 1992;85:1792-1798.

24. Briel M, Ferreira-Gonzalez I, You JJ, Karanicolas PJ, AkI EA, Wu P, Blechacz B, Bassler D, Wei X, Sharman A, Whitt I, Alves da Silva S, Khalid Z, Nordmann AJ, Zhou Q, Walter SD, Vale N, Bhatnagar N, O’Regan C, Mills EJ, Bucher HC,
Montori VM, Guyatt GH. Association between change in high density lipoprotein cholesterol and cardiovascular disease morbidity and mortality: systematic review and meta-regression analysis. BMJ. 2009;338:b92.

25. Podrez EA. Anti-oxidant properties of high-density lipoprotein and atherosclerosis. Clin Exp Pharmacol Physiol. 2010;37:719-725.

26. Nofer JR, Brodde MF, Kehrel BE. High-density lipoproteins, platelets and the pathogenesis of atherosclerosis. Clin Exp Pharmacol Physiol. 2010;37:726735.

27. FitzGerald GA, Pedersen AK, Patrono C. Analysis of prostacyclin and thromboxane biosynthesis in cardiovascular disease. Circulation. 1983;67: 1174-1177.

28. Patrono C, Falco A, Davì G. Isoprostane formation and inhibition in atherothrombosis. Curr Opin Pharmacol. 2005;5:198-203.

29. Pignatelli P, Carnevale R, Di Santo S, Bartimoccia S, Sanguigni V, Lenti L, Finocchi A, Mendolicchio L, Soresina AR, Plebani A, Violi F. Inherited human gp91phox deficiency is associated with impaired isoprostane formation and platelet dysfunction. Arterioscler Thromb Vasc Biol. 2011;31:423-434.

30. Pratico D, Lawson JA, FitzGerald GA. Cyclooxygenase-dependent formation of the isoprostane, 8-epi prostaglandin F2 alpha. J Biol Chem. 1995;270:9800-9808.

31. Camarillo-Romero E, Dominguez-Garcia MV, Amaya-Chavez A, CamarilloRomero Mdel S, Talavera-Piña J, Huitron-Bravo G, Majluf-Cruz A. Effects of a physical activity program on markers of endothelial dysfunction, oxidative stress, and metabolic status in adolescents with metabolic syndrome. ISRN Endocrinol. 2012;2012:970629.

32. Kim JY, Kim OY, Paik JK, Kwon DY, Kim HJ, Lee JH. Association of age-related changes in circulating intermediary lipid metabolites, inflammatory and oxidative stress markers, and arterial stiffness in middle-aged men. Age (Dordr). Jul 18, 2012. doi:10.1007/s11357-012-9454-2. Available at http:// link.springer.com. Accessed March 28, 2013.

33. Patrono C, Andreotti F, Arnesen H, Badimon L, Baigent C, Collet JP, De Caterina R, Gulba D, Huber K, Husted S, Kristensen SD, Morais J, Neumann FJ, Rasmussen LH, Siegbahn A, Steg PG, Storey RF, Van de Werf F, Verheugt F. Antiplatelet agents for the treatment and prevention of atherothrombosis. Eur Heart J. 2011;32:2922-2932. 


\section{Journal of the American Heart Association \\ OPEN ACCESS f}

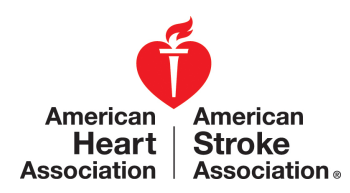

\section{Enhanced Lipid Peroxidation and Platelet Activation as Potential Contributors to Increased Cardiovascular Risk in the Low-HDL Phenotype}

Natale Vazzana, Antonina Ganci, Angelo Baldassare Cefalù, Stefano Lattanzio, Davide Noto, Nicole Santoro, Raoul Saggini, Luca Puccetti, Maurizio Averna and Giovanni Davì

J Am Heart Assoc. 2013;2:e000063; originally published April 4, 2013; doi: 10.1161/JAHA.113.000063

The Journal of the American Heart Association is published by the American Heart Association, 7272 Greenville Avenue, Dallas, TX 75231

Online ISSN: 2047-9980

The online version of this article, along with updated information and services, is located on the World Wide Web at:

http://jaha.ahajournals.org/content/2/2/e000063 